\title{
Auxins and Cytokinins in Plant Development 2005
}

\author{
Miroslav Kamínek, ${ }^{1}$ Jutta Ludwig-Müller, ${ }^{2}$ Radomíra Vaňková, ${ }^{1}$ and \\ Eva Zažímalová ${ }^{1 *}$ \\ ${ }^{1}$ Institute of Experimental Botany, ASCR, Rozvojova 135, 16502 Prague 6, Czech Republic; ${ }^{2}$ Institut für Botanik, Technische \\ Universität Dresden, 01062 Dresden, Germany
}

\section{INTRODUCTION}

The International Symposium "Auxins and Cytokinins in Plant Development 2005" (ACPD 2005), held in Prague, Czech Republic, July 7-12, 2005 , was well timed to reflect the current enormous progress in research of the two classes of phytohormones. The symposium was a continuation of a long tradition of conferences devoted to plant growth substances organized by the Institute of Experimental Botany of the Academy of Sciences of the Czech Republic. Five international symposia have already been held in the baroque chateau at Liblice near Prague, and the sixth and last one, "Auxins and Cytokinins in Plant Development 1999" was held in Prague itself.

The ACPD 2005 meeting provided a platform for discussions on recent achievements and future directions in and prospects for research on the two hormones for controlling plant development. The meeting fulfilled expectations and highlighted the very recent advances in molecular biology, biochemistry, and physiology of both phytohormones,

*Authors are listed alphabetically

Received 17 October 2005; accepted 18 October 2005; Online publication 28 February 2006

*Corresponding author ; e-mail: eva.zazim@ueb.cas.cz as well as some aspects of their applications in biotechnology. The scientific program consisted of two opening lectures and seven sessions, which always included both hormones: (1) Biosynthesis and metabolism, (2) Transport, (3) Signaling, (4) Pattern formation and development, (5) Auxin-cytokinin crosstalk and interaction with other hormones, (6) Responses to environment, and (7) Novel methods. The interplay of these processes during plant development is shown in Figure 1. The conference attracted 180 participants from 28 countries in Europe, North America, Asia, Africa, and Australia. Abstracts of oral and poster presentations were published in Biologia Plantarum 49 (Suppl.) 2005 (and are also available on the Web at http://acpd. cas.cz). A comparison between the proportion of contributions to the topics selected for the 1999 conference with that in 2005 revealed that most of the topics selected in 2005 received even attention by the participants, reflecting the attractive program of the meeting (Figure 2). Most noticeable was the increase in contributions in the transport session in 2005 compared to 1999.

In the first plenary lecture which he dedicated to Prof. Milan Kutáček, Athanasios Theologis (Plant Gene Expression Center, Albany, NY, USA) focused on the native auxin, indole-3-acetic acid, as a "simple 


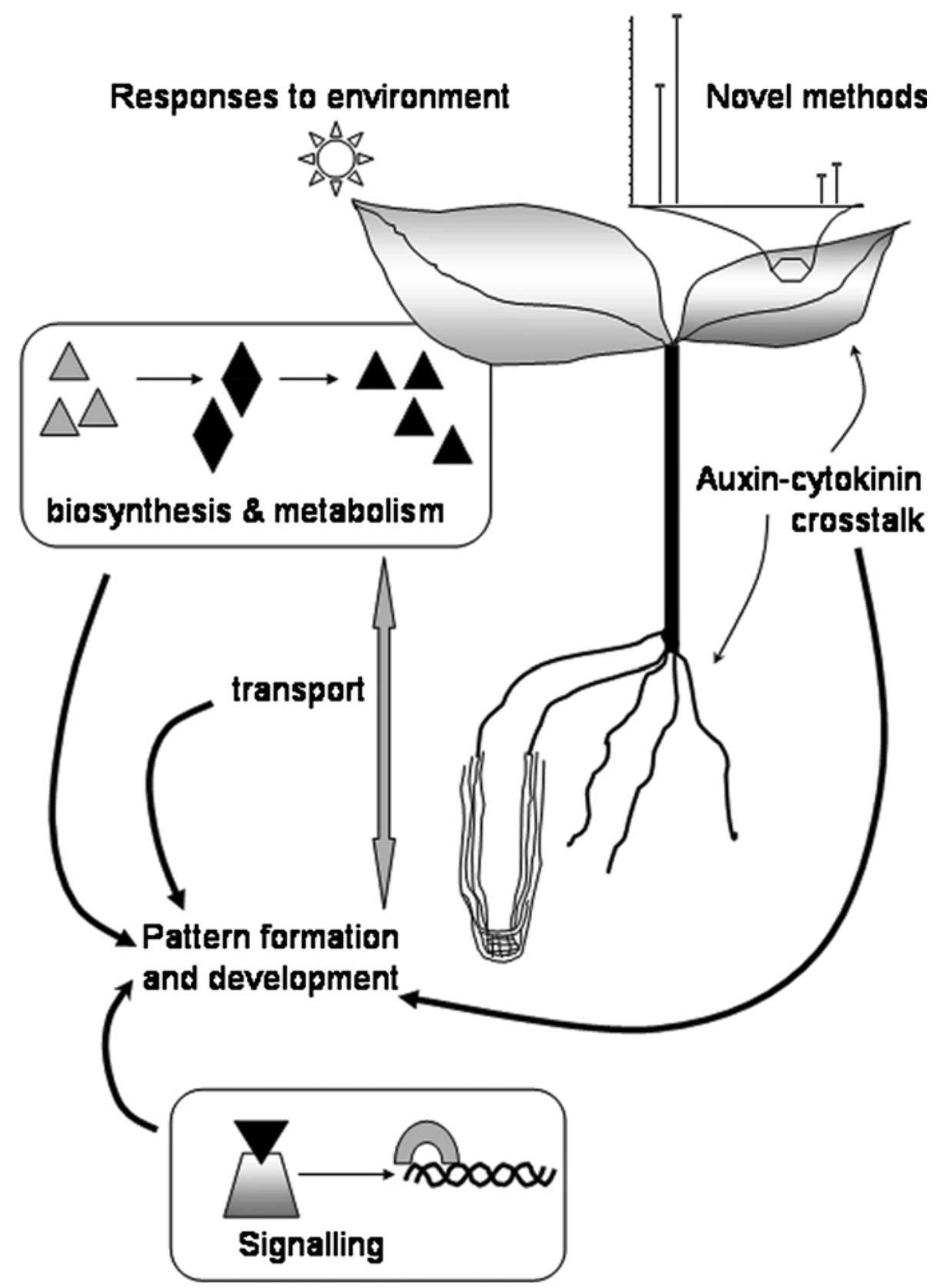

Figure 1. The ACPD 2005 conference covered seven topics, which were presented in oral and poster forms. The topics are shown in relation to their function in the plant. Possible interactions between biosynthesis/metabolism, transport, signaling, and pattern formation are also shown.

molecule with complex reputation." He gave an overview of the history of research on this pleiotropic signaling molecule and connected it with the present state of the art and future prospects, with emphasis on transcriptional regulation.

The second plenary lecture was presented by Thomas Schmülling (Free University of Berlin, Germany), who summarized recent progress in identification of genes encoding cytokinin biosynthetic (isopentenyltransferases) and degradative enzymes (cytokinin oxidase/dehydrogenases, CKX), as well as cytokinin receptors, phosphotransmitters, and response regulator proteins.

\section{Biosynthesis and Metabolism}

Two contributions in this session focused on auxin metabolism: Bonnie Bartel (Rice University, Houston, Texas, USA) presented evidence for the 


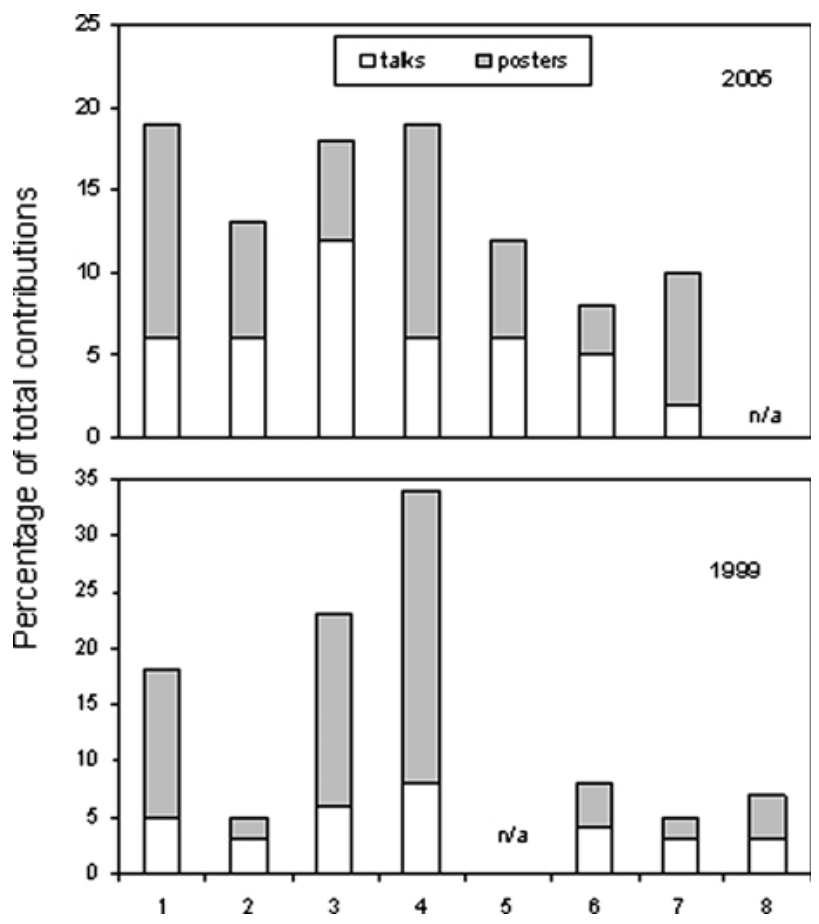

Figure 2. The number of contributions (talks or posters) on the different topics from the ACPD 2005 meeting in comparison to the topical contributions in 1999. n/ $\mathrm{a}=$ topic was not represented at this meeting. (1) Biosynthesis and metabolism, (2) Transport, (3) Signaling (includes gene expression), (4) Pattern formation and development (development/reproduction in 1999), (5) Auxin-cytokinin crosstalk and interaction with other hormones (only at ACPD 2005), (6) Responses to environment, (7) Novel methods, (8) Cell cycle (only at the 1999 conference).

involvement of IAA amino acid conjugate hydrolases in auxin homeostasis in Arabidopsis. She described the screening of additional IAA conjugate-resistant mutants and the identification of the mutated genes. In addition, indole-3-butyric acid (IBA) conversion to IAA in peroxisomes was described in detail and the possible function of IBA as auxin was discussed. Jutta Ludwig-Müller (Technische Universität, Dresden, Germany) showed data on the activity of auxin conjugate hydrolases from different plant species and discussed these findings with respect to the evolution of auxin homeostasis.

The remainder of the session was devoted to cytokinins. Hitoshi Sakakibara (RIKEN, Yokohama, Japan) described the hydroxylation of isopentenyladenine-type cytokinins by cytochrome P450 mono-oxygenases CYP735Al and CYP735A2 as a key step of trans-zeatin biosynthesis. The expression of these enzymes is cytokinin-responsive and organ- specific. Root-specific induction of CYP735A2 expression by cytokinins indicates that transhydroxylation might be involved in regulation of cytokinin metabolism and signaling in roots. It seems that xylem cytokinins carry a long-distance nutritional signal, whereas phloem cytokinins function as signals for sink strength regulation. Norbert Brugière (Pioneer Hi-Bred International, Johnson, Iowa, USA) reported the isolation and characterization of the maize cytokinin biosynthesis gene (ZmIPT2), which is expressed in kernels, where it controls cytokinin levels together with CKX and regulates sink strength. Machteld Mok (Oregon State University, Corvallis, Oregon, USA) described the structural similarity of trans-zeatin and $m$-topolin (3-hydroxybenzyladenine), as well as of ciszeatin, o-topolin (2-hydroxybenzyladenine), and thidiazuron, which was confirmed by comparison of their affinity for bean trans-zeatin $O$-glucosyltransferase ZOGl and maize cis-zeatin $O$-glucosyltransferase, respectively, and correlated it with their affinity for cytokinin receptors AHK4 and ZmHKl. Radka Fohlerová (Institute of Biophysics, Brno, Czech Republic) presented a functional analysis of the active site of the maize $\beta$-glucosidase Zm-p60.1 specific for cytokinin-O-glucosides. Ivo Frébort (Palacký University, Olomouc, Czech Republic) gave a thorough kinetic analysis of CKX isoenzymes using different cytokinin substrates, which indicated the formation of a binary ES complex of cytokinin imine and the reduced form of the enzyme. He found that benzyladenine and kinetin can form stable complexes with the enzyme, thus blocking degradation of endogenous cytokinins. Marta Fernandez Núñez (Universität Hamburg, Germany) described CKX overexpression in the moss Physcomitrella patens resulting in reduced cell number and decreased bud formation. Interestingly, in addition to cytokinin bases, cytokinin ribotides, which are not substrates of the enzyme, were also decreased.

\section{Posters in the Biosynthesis and Metabolism Session}

Posters were devoted to evaluating the role of different CKX isoenzymes and identifying specific enzyme amino acids essential for their activity (I. Bartrina and others, Free University of Berlin, Germany), cloning of CKX2 in yeast and characterizing biochemical properties of the excreted enzyme (J. Frébortová and others, Institute of Experimental Botany, Olomouc, Czech Republic), elucidating the expression patterns of different maize CKX genes during ontogenesis of different organs and tissues 
(N. Houba-Hérin and others, INRA, Versailles, France), characterizing the crystal structure of maize CKXI (D. Kopečný and others, Palacký University, Olomouc, Czech Republic/INRA, Versailles, France), and the stimulation of CKX activity in the early stages of leaf senescence in cereals (P. Galuszka and others, Palacký University, Olomouc, Czech Republic). Other posters were focused on the role of two cytochrome P450 mono-oxygenases in the maintenance of cytokinin homeostasis (H.B. Kim and others, Seoul National University, Seoul, Korea) and the control of fruit and lateral root development (C. J. Oh and others, Seoul National University, Seoul, Korea), root vascular tissue and flower bud development (K. Takei and H. Sakakibara, RIKEN, Yokohama, Japan), on the impact of bean transzeatin O-glucosyltransferase overexpression under the control of the ubiquitin promoter on maize phenotype (A. Pineda Rodo and others, Oregon State University, Corvallis, Oregon, USA), regulated expression of isopentenyltransferase on cytokinin metabolism, nitrogen uptake, and allocation in wheat (B. Šolcová and others, Institute of Experimental Botany, Prague, Czech Republic), detection of a third putative N-glucosyltransferase in Arabidopsis by activation tagging (S. Dwivedi, University of Connecticut, Storrs, Connecticut, USA, and others), and regulation of cytokinin metabolism in pea by CKX and zeatin reductase (A. Gaudinová and others, Institute of Experimental Botany, Prague, Czech Republic). Interesting results were provided in presentations about the intracellular localization of zeatin $O$-glucosides in vacuoles (N. S. Kiran and others, Masaryk University, Brno, Czech Republic), the effect of high doses of cytokinin ribosides and bases on reactive oxygen species evolution and apoptosis initiation (P. Mlejnek and others, Mendel University, Brno, Czech Republic), comparison of the effect of different auxins on shoot differentiation in Atriplex nummularia (A. S. Al-Wasel, Al-Quassim University, Al-Quassim, Saudi Arabia), determination of the level of auxin and its conjugates in different root cell types using a novel technique-Fluorescence Activated Cell Sorting (S. V. Petersson and others, Umeå Plant Science Center, Umeå, Sweden) and auxin and cytokinin biosynthesis in rhizosphere bacteria including both endosymbionts and pathogens (N. B. Zdanowska and E. Prinsen, University of Antwerp, Wilrijk, Belgium). Plasmodiophora infection was found to be connected in Arabidopsis with stimulation of auxin conjugation to amino acids by the products of the GH3-gene family (C. Horn and others, Botany Institute, TU Dresden, Germany).

\section{TrANSPORT}

Because there are very limited data in the literature about mechanism(s) of cytokinin transport, this session was almost entirely concentrated on transport of auxins. The papers presented reflected the fact that at present there are two main candidates for direct involvement in the efflux of auxin molecules from cells, that is, PIN-type and MDR-type proteins.

In the session-opening talk, Jiří Friml (University of Tübingen, Germany) compared the present state of the art in the auxin transport field with what was known in 1999 when the previous ACPD meeting was held, and stressed the major progress achieved. Then he summarized the present knowledge about auxin efflux facilitators from the PIN protein family and their major role in a network underlying the formation of local temporal auxin gradients necessary for plasticity and adaptability of plant development. Christian Luschnig (University of Natural Resources and Applied Life Science-BOKU, Vienna, Austria) focused on the cell-specific degradation of the root-specific PIN2/EIR1/AGR protein in relation to gravity stimulus. He showed that the gravity response of Arabidopsis roots involved not only PIN2-mediated auxin transport but also proteasome-dependent degradation of PIN2. Remko Offringa (Leiden University, The Netherlands) described the role of PINOID (PID) protein kinase in the PINOID (PID)-dependent binary switch controlling the polar localization of PINs, and the role of two calcium-binding proteins in the PID-signaling cascade. Eva Zažímalová (Institute of Experimental Botany, Prague, Czech Republic) showed that the "semi-heterologous" overexpression of AtPINs in tobacco cells increased auxin efflux from cells and resulted in phenotypic changes that were typical for auxin depletion; the data suggested a rate-limiting role of PINs in auxin efflux.

In the second part of the session Angus Murphy (Purdue University, West Lafayette, Indiana, USA) highlighted in his overview talk the role of plant orthologs of human $\mathrm{ABC}$ transporters from the family of multiple drug resistance/P-glycoproteins (MDRs/PGPs) in auxin transport in plant cells. He summarized the recent data on expression in plant, yeast, as well as human cells, and suggested a possible co-action of these proteins with some PINs in the control of auxin transport. Markus Geisler (University of Zurich, Switzerland) characterized the twisted dwarfl (TWD1), an immunophilin-like protein that interacts with $\mathrm{ABC}$ transporters PGP1 and PGP19 involved in auxin transport. TWD1 may regulate the auxin transport activity of PGPs. 
Tom Bennett (University of York, UK) reported the characterization and cloning of ANTIMAXl (ATM1), which encodes a phospholipase 2 modulating the auxin transport activity, and consequently controlling the branching phenotype. Momoyo Ito (University of Tokyo, Japan) introduced a new mutant of rice, the fish bone (fib), exhibiting pleiotropic abnormalities in most plant organs. Mutants fib also showed impaired polar auxin transport activity.

\section{Posters in the Transport Session}

Posters were devoted to the pharmacological approach of studying auxin redistribution (D. Crouch, Institut des Sciences du Vegetal, Gif sur Yvette, France), the computational modeling of long-distance auxin transport (C. Beveridge and others, University of Queensland, Brisbane, Australia), characterization of equilibrative nucleoside transporter OsENT2 and its function in cytokinin transport (N. Hirose and others, RIKEN Plant Science Center, Yokohama, Japan), the mechanism for accumulation of cytokinins in tobacco cells (P. Klíma and others, Institute of Experimental Botany, Prague, Czech Republic), the possible role of PaLAXI as an auxin influx carrier (K. Hoyerová and others, Institute of Experimental Botany, Prague, Czech Republic), the characterization of tobacco cell lines transformed with the PaLAXl gene (M. Laňková and others, Institute of Experimental Botany, Prague, Czech Republic) or with the AtPIN1, 4, 6, and 7 genes (P. Skůpa and others, Institute of Experimental Botany, Prague, Czech Republic), the roles of flavonoids in auxin transport, signaling, trafficking, and reactive oxygen species scavenging (W. A. Peer and A. S. Murphy, Purdue University, West Lafayette, Indiana, USA), preparation of Arabidopsis cell lines for studying auxin transport (D. Seifertová and others, Institute of Experimental Botany, Prague, Czech Republic), and the auxin-mediated crossregulation of PIN expression (A. Vieten and others, University of Tübingen, Germany).

\section{SigNALING}

Tom Guilfoyle (University of Missouri, Columbia, Missouri, USA) in the session-opening talk gave an overview of the function of auxin response factors (ARFs) and Aux/IAA transcriptional repressors. The interaction between ARFs and auxin response elements (AuxREs) in promoters of auxin response genes, as well as the association of Aux/IAAs with ARF activators was characterized and related to the response to hormones and environment. Bekele
Abebie (The Hebrew University of Jerusalem, Rehovot, Israel) showed that different levels of Aux/ IAA genes might be involved in regulation of abscission in Cestrum elegans.

Joe Kieber (University of North Carolina, Chapel Hill, North Carolina, USA) in the second overview talk of this session reported on the functional redundancy of the two-component cytokinin signaling system (especially of histidine-containing phosphotransmitters AHPs and type-A response regulators). ARR5 protein was stabilized by cytokinins via two-component signaling by phosphorylation, otherwise being readily degraded by the proteasome pathway. Jana Hradilová (Institute of Biophysics, Brno, Czech Republic) stressed the high organ specificity of AHP1 (mainly in roots), AHP2 (mainly in flowers), and AHP4. Expression of AHP3 and AHP5 appeared to be more uniform. Both exogenous and elevated endogenous cytokinins stimulated AHP1-4 expression in short-term treatment (1-24 h). Michael Riefler (Free University of Berlin, Germany) presented results of phenotypical analysis indicating partially overlapping functions of three cytokinin receptors. Triple receptor mutants exhibited increased cytokinin levels. Georgy Romanov (Institute of Plant Physiology, Moscow, Russia) compared ligand specificity of cytokinin receptors AHK3 and AHK4. Both had the highest affinity towards trans-zeatin; however, they differed in the affinity to isopentenyladenine (higher in case of AHK4) and dihydrozeatin. Jan Barciszewski (Polish Academy of Sciences, Poznan, Poland) presented an $x$-ray crystallography study of the cytokinin complex with lectin and in silico modeling of the cytokinin receptor AHK4. Ildoo Hwang (Pohang University, Korea) reported that AHK3 was receptor specific for leaf longevity control. Phosphorelay from AHK3 to the conserved Asp80 residue of ARR2 rendered degradation of ARR2 via proteasome. Stabilization of ARR2 led to the delay of senescence. $\mathrm{Ni} \mathrm{Ni}$ Tun (University of Hannover, Germany) described rapid stimulation of nitric oxide (NO) biosynthesis by cytokinins within 2 minutes. NO synthase was not involved in NO synthesis in plants. The potential role of NO in mediation of plant responses to zeatin was discussed.

Mark Estelle (Indiana University, Bloomington, Indiana, USA) summarized knowledge on the auxin signaling pathway via ubiquitin ligase $\mathrm{SCF}^{\mathrm{TIR} 1}$ mediated control of transcription. He showed that TIR 1, as well as three other F-box proteins, AFB 1, 2, 3 , function as auxin receptors and collectively mediate control of gene expression by auxin via ubiquitin-dependent protein degradation. These findings were supported in the talk given by Stefan 
Kepinski (University of York, UK), and the direct binding of auxin to TIRl was shown, as was the modification of the $\mathrm{SCF}^{\mathrm{TIR} 1}$ complex.

Richard Napier (Warwick HRI, University of Warwick, UK) gave a comprehensive overview of gene-expression-independent auxin signaling via the auxin binding protein $1(\mathrm{ABPl})$ and reported on the present understanding of the physicochemical nature of the binding of the auxin molecule to the ABPl protein. Hartwig Lüthen (University of Hamburg, Germany) presented data confirming the relevance of ABP1 to growth-related processes and suggesting the involvement of DGT (diageotropica) in the auxin signaling cascade downstream from ABP1. Marie Shishova (St. Petersburg State University, Russia) used maize coleoptiles and tobacco cells to investigate sensitivity to auxin in relation to growth, and suggested that the loss of plant cell sensitivity to auxin within the elongation process depends on decreased amounts of ABP1 in the cells. A novel mode of hormone action-the effect of auxin on protein trafficking-was introduced by Michael Sauer (University of Tübingen, Germany), and a possible role for ABPl in its mechanism was suggested.

\section{Posters in the Signaling Session}

Posters concentrated on: the characterization of the tomato Aux/IAA-type gene LeIAA3 (Chaabouni and others, INRA/INP Toulouse, France), the role of ABP4 in auxin and light signaling in maize (M. Fellner, Palacký University, Olomouc, Czech Republic, and others), characterization of cytokinin responses in Arabidopsis cell suspension culture (Chakrabortee and Murray, University of Cambridge, UK), protein-protein interaction in the cytokinin signaling pathway in A. thaliana (A. Heyl, Free University of Berlin, Germany, and others), changes in cytoplasmic calcium signaling after treatment with auxin, cytokinin, and galactoglucomannan oligosaccharides (A. Kákošová, Institute of Chemistry, Bratislava, Slovakia, and others), the role of phospholipase C and D, and calcium ions in cytokinin signal transduction (V. S. Kravets, Institute of Bioorganic Chemistry and Petroleum Chemistry, Kiev, Ukraine, and others, and S. Medvedev, University of St. Petersburg, Russia, and others, respectively); the ligand specificity of cytokinin receptors from Arabidopsis and the cytokinin activity of various 6-benzylaminopurines (G. A. Romanov, Institute of Plant Physiology, Moscow, and others, and L. Spíchal, Palacký University, Olomouc, Czech Republic, and others, respectively).

\section{Pattern Formation and Development}

In her session-opening talk Ottoline Leyser (University of York, UK) summarized both "classical" (auxins and cytokinins) and more recent components of the signaling network controlling shoot branching. MAXl to MAX4 may define an additional branch-inhibiting pathway, downstream of and independent of auxin. Three of these loci seem to be involved in the production of a new hormone that inhibits bud growth. Christine Beveridge (University of Queensland, Brisbane, Australia) described $R A$ MOSUS genes RMS1 and RMS5, which control levels of an as yet unidentified mobile branching inhibitor. This inhibitor is involved in a complex network with auxins and cytokinins, regulating shoot branching in pea. A complex shoot-to-root-to-shoot feedback sytem was presented. Boyeong Ha Lee (Cold Spring Harbor Laboratory, Cold Spring Harbor, New York, USA) reported that phyllotaxy is controlled by $\mathrm{ABPH}$, a type-A response regulator. In a feed-backlike control mechanism ABPHl represses cytokinininduced expansion of shoot apical meristem, which results in an alternating leaf pattern. ABPHl is localized in the nucleus, and the inhibition of auxin polar transport reduces ABPHl expression. Philip Brewer (University of Tübingen, Germany) showed auxin distribution and PIN proteins in relation to flower phyllotaxy and stressed the involvement of other PINs in addition to PINl.

Malcom Bennett (University of Nottingham, UK) gave a comprehensive review of the role of polar auxin transport in lateral root initiation and development in his overview talk, and he suggested the involvement of negative cytokinin regulation on the level of xylem-pole pericycle cells, but not young lateral root primordia, in the lateral root development in Arabidopsis.

Ari Pekka Mähönen (University of Helsinki, Finland) showed that the wol (woodenleg) mutant (lacking functional cytokinin receptor WOL/AHK4) has a reduced number of provascular cell files, which all differentiate into xylem. He identified SOWl, acting downstream of AHK4, as a negative regulator of cytokinin signaling and cell proliferation. Thomas Roitsch (University of Würzburg, Germany) reported that the cytokinin effect on the delay of senescence is mediated by the stimulation of extracellular invertase activity. Extracellular cleavage of the transport sugar sucrose into hexose monomers is essential for assimilate partitioning and sink strength. The involvement of auxin response factors (ARF3, 4) in the modulation of KANADI activity in control of Arabidopsis organ 
polarity was shown by Yuval Eshed (Weizman Institute of Science, Rehovot, Israel).

\section{Posters in the Session Pattern Formation and Development}

Posters were devoted to cellular and organogenic responses of Arabidopsis pericycle to auxins and cytokinins (D. Chriqui and others, University of P. \& M. Curie, Paris, France), control of root meristem growth in Arabidopsis by cytokinin (S. Sabatini, University "La Sapienza," Rome, Italy, and others), the molecular characterization of MAXl (T. Sieberer, University of York, UK), polar auxin transport in pea axillary buds in relation to apical dominance (J. Balla, Mendel University of Agriculture and Forestry, Brno, Czech Republic, and others), apical meristem development after transcriptional activation of IPT in Arabidopsis (J. Dubová, Masaryk University, Brno, Czech Republic), cytokinin control of expression of KNOTTEDl-like homeobox genes during shoot development in Arabidopsis (P. Souček, Institute of Biophysics, Brno, Czech Republic, and others), activation of cytokinin biosynthesis by class 1 homeobox (KNOXl) genes in Arabidopsis (N. Oria, The Hebrew University of Jerusalem, Rehovot, Israel, and others), overexpression of the IPT gene in developing Arabidopsis seedlings carrying auxin reporters (A. Kuderová and B. Brzobohatý, Masaryk University, Brno, Czech Republic), functional characterization of CKX in Arabidopsis (T. Werner and others, Free University Berlin, Germany), cytokinin levels, CKX and $\beta$-glucosidase activities in tobacco leaf segments during organogenesis in vitro (Z. Prokešová, Mendel University of Agriculture and Forestry, Brno, Czech Republic, and others), cytokinin level and CKX activity in pea in relation to developmental senescence (I. Vaseva-Gemisheva, Institute of Plant Physiology, Sofia, Bulgaria), the role of cytokinins in potato tuber development (J. Malbeck, Institute of Experimental Botany, Prague, Czech Republic, and others), tuber formation, cytokinin content, and carbohydrate metabolism in transgenic potato plants carrying rolC and invertase genes (E. V. Grishunina, Institute of Plant Physiology, Moscow, Russia, and others), manipulation of meristem function by local manipulation of cell cycle parameters (J. Wyrzykowska, ETH, Zurich, Switzerland, and A. Fleming), the role of cytokinins in antioxidative mechanisms in tobacco leaves during ageing ( $\mathrm{Z}$. Mýtinová, Charles University, Prague, Czech Republic, and others), epigenetic modifications associated with 2,4-D use during oil palm somatic embryogenesis (T. J. Tranbarger, IRD/CIRAD Palm Biology Lab., Montpellier, France), regulation of flowering in the vine cacti (A. Khaimov, University of the Negev, Beer-Sheva, Israel), and the effects of growth regulators on somatic embryogenesis in Drosera rotundifolia (E. Hlinková, Comenius University, Bratislava, Slovakia, and others).

\section{Auxin-Cytokinin Crosstalk and INTERACTION WITH OTHER HORMONES}

In the session-opening talk, Goran Sandberg (Umeå Plant Science Center, Sweden) described the primary root tip and lateral root tips as important sources of auxin. Auxin synthesis in root tips is under rapid positive control of cytokinins. To find out in which tissues auxin synthesis occurs, Arabidopsis plants expressing GFP constitutively in different root cell types were employed. After protoplast isolation, individual cell types were separated using FACS (Fluorescence Activated Cell Sorting). IAA levels were significantly higher in the stele than in the endodermis and lateral root cap.

Hitoshi Mori (Nagoya University, Japan) described the mechanism of apical dominance repression. After decapitation, a rapid increase in the expression of cytokinin biosynthetic genes PsIPT1 and PsIPT2 in nodal stem tissue was found. The promoter region of PsIPT2 was repressed by IAA. Thus locally synthesized cytokinins, rather than root-derived ones, seem to be involved in the outgrowth of the axillary bud. Jan Hejátko (Masaryk University, Brno, Czech Republic) reported the role of CKIl, sensor histidin kinase, in megagametogenesis. CKIl ectopic expresssion overlapped with auxin intracellular maxima. Regulation of CKII expression in roots by both cytokinin and auxin provides molecular evidence for hormone interplay during Arabidopsis root development. Ivana Macháčková (Institute of Experimental Botany, Prague, Czech Republic) compared the levels of ABA and IAA in leaves and chloroplasts of plants with manipulated cytokinin levels. The data suggested that modification in the level of cytokinins affects not only IAA but also ABA levels. Sunghwa Choe (Seoul National University, Korea) analyzed the brassinosteroid response in Arabidopsis dwarf mutants and showed that BES1, a member of the brassinosteroid signal transduction chain, modulates Aux/IAA genes as well as a cytochrome P450 involved in brassinosteroid synthesis. Harry Van Onckelen (University of Antwerp, Belgium) gave an extensive overview on the role of cytokinins in the control of cell cycle progression. Whereas transzeatin is indispensable for $G 2 / M$ transition, a cytokinin requirement for cell cycle initiation and termination is less specific. Eva Benková (ZMBP, 
University Tübingen, Germany) talked about hormonal regulation of lateral root development and showed that although cytokinins directly inhibited lateral root initiation, the inhibition of root growth was mediated via ethylene. Hanne Rasmussen (KVL, Hoersholm, Denmark) followed the dynamics of levels of IAA and cytokinins in relation to seasonal and spatial aspects of development of Abies nordmanniana Spach. John Chandler (University of Cologne, Germany) studied embryonic patterning in Arabidopsis and found that DORNROESCHEN/ENHANCER of shoot regenerationl (DRN/ESR l) and DRN-like proteins, which are positively regulated by auxin, interact with class III HD-ZIP proteins and control embryo patterning.

\section{Posters in the Session Auxin-Cytokinin Crosstalk and Interaction with Other Hormones.}

Posters were focused on: the role of auxins and cytokinins in clubroot disease in Arabidopsis (J. Ludwig-Müller, Technische Universität Dresden, Germany, and others), auxin-cytokinin crosstalk in petunia during fertilization (L. Kovaleva, Institute of Plant Physiology, Moscow, and others), altered auxin and cytokinin responses in ramosus branching mutants of pea (N. Young and C. Turnbull, Division of Biology, Imperial College London, Wye, UK), the role of galactoglucomannan oligosaccharides and their co-action with auxins in elongation growth (K. Kollárová and others, Institute of Chemistry, Bratislava, Slovakia), positive regulation of auxindependent lateral root initiation by jasmonic acid in Arabidopsis (A. Swiatek and others, University of Atwerp, Belgium), various interactions between cytokinins and ABA (J. Pospíšilová, Institute of Experimental Botany, Prague, Czech Republic), CKX activity as a tool in gibberellin/cytokinin crosstalk (I. Vaseva-Gemisheva, Institute of Plant Physiology, Sofia, Bulgaria, and others), auxin and gravity-dependent expression of members of a zinc finger "ID domain" protein family in rice (C. Han, Gyeongsang National University, Jinju, South Korea, and others), and the role of cytokinins in programmed cell death in tobacco cells (O. Smetana, Charles University, Prague, Czech Republic).

\section{RESPONSES TO ENVIRONMENT}

In the session-opening talk, Johannes van Staden (University of KwaZulu-Natal, Pietermaritzburg, South Africa), outlined the flow and "recycling" of cytokinins in the environment. The level of cytokinins in the soil should be considered because of multiple soil-borne microorganisms (for example, mycorrhiza and soil microalgae), decomposing leaves, as well as cytokinin excretion from plants.

Jean Yong (Australian National University, Australia) described the effect of cytokinin overproduction on leaf physiology and the response to $\mathrm{CO}_{2}$ enrichment in potato. Bigger stems and more trichome formation were found in IPT overexpressing plants. Stomata were more open during the day, which led to elevated photosynthesis rates (under high humidity and moderate light). Radomíra Vaňková and others (Institute of Experimental Botany, Prague, Czech Republic) compared the drought response of tobacco plants with modified cytokinin metabolism. They found that considerably elevated cytokinin levels inhibited adaptation of the root system to drought, whereas mild cytokinin increases improved subsequent recovery of plants. Vladislav Yemelyanov (St. Petersburg State University, Russia) compared the effects of anoxia on growth and on ABA and IAA levels in wheat and rice. Better seedling survival was achieved after pretreatment with exogenous ABA and IAA. Roni Aloni (Tel Aviv University, Israel) explained cytokinin inhibition of lateral root formation as "root apical dominance," which resulted in stimulation of main root elongation. He stressed the significance of the transpiration stream in translocation of rootborne cytokinins to the shoots; cytokinins accumulated around opened stomata and diffused away when stomata closed. The relationship between IAA biosynthesis and host-derived flavonoids in relation to symbiosis was reported by Els Prinsen (University of Antwerp, Belgium) in Rhizobia. Eugeni Ananiev (Institute of Plant Physiology, Sofia, Bulgaria) observed that benzyladenine can increase RNA polymerase in Cucurbita cotyledons.

\section{Posters in the Session Responses to Environment}

Posters dealt with the effect of salinity on cytokinin levels (L. Atanasova, Institute of Plant Physiology, Sofia, Bulgaria, and others), modulation of cytokinin content and CKX activity in response to temperature (I. Vaseva-Gemisheva, Institute of Plant Physiology, Sofia, Bulgaria, and others), cytokinin content during senescence and in response to jasmonate (K. Ananieva, Acad. M. Popov Institute of Plant Physiology, Sofia, Bulgaria, and others), and the delay of chlorophyll degradation after treatment with cytokinins (M. Sedlárová, Palacký University, Olomouc, Czech Republic, and others). 


\section{Novel Methods}

The session opened with a talk by Miroslav Strnad (Institute of Experimental Botany, Olomouc, Czech Republic) in which he described recent progress in techniques for auxin and cytokinin quantification, mainly the development of GC-MS and LC-MS methods. The specificity and detection limits have been improved by the combination of immunoaffinity chromatography and triple quadrupole/Q-Tof MS. A new trend, the use of ultraperformance liquid chromatography, was also presented. Gabriela Bohmová (Masaryk University, Brno, Czech Republic) described proteome analysis (protein extraction, 2Delectrophoresis, programs for spot analysis and MS identification) using the example of identifying cytokinin-induced proteins in dark-grown Arabidopsis seedlings. Klára Hoyerová (Institute of Experimental Botany, Prague, Czech Republic) presented a comparative study of different extraction and purification techniques for cytokinin analysis.

\section{Posters Describing Novel Methods}

Posters were devoted to: the new heavy-labeled auxins for mass spectrometry (R. Buchtík, Institute of Experimental Botany, Olomouc, Czech Republic, and others), analysis of cytokinins in coconut using capillary electrophoresis (J. H. W. Yong, Nanyang Technological University, Singapore), comparison of different procedures for the extraction of plant growth substances for analysis using LC-MS/MS (B. Veierskov, KVL, Frederiksberg, Denmark, and others), the immunomagnetic separation method for isolation of new cytokinin derivatives (E. Hauserová and others, Palacký University, Olomouc, Czech Republic), new chromatographic and mass spectrometric approaches for cytokinin analysis (O. Novák and others, Palacký University, Olomouc, Czech Republic), immunoaffinity extraction in auxin analysis (K. Sedláková, Palacký University, Olomouc, Czech Republic, and others), a new sensitive method for enzyme kinetic study of rare glucosides (P. Mazura, Masaryk University, Brno, Czech Republic, and others), quantitation of gene expression under drought (H. Štorchová and others, Institute of Experimental Botany, Prague, Czech Republic), and the effect of elevated cytokinins on the Arabidopsis thaliana proteome (P. Váňová, Masaryk University, Brno, Czech Republic). The development of a Fluorescence Resonance Energy Transfer (FRET) biosensor for phytohormones (G. O. Badescu, Warwick HRI, University of Warwick, UK, and others) will open a new field in plant hormone analysis.

\section{Conclusions}

The symposium was informative, and the papers presented reflected the very recent achievements in the fields of both auxins and cytokinins. Comparison with the ACPD meeting in 1999 showed tremendous progress in the state of the art, especially in the biosynthesis of both hormones, the mechanism of polar auxin transport (the role of PINs in plant development; involvement of MDRs/PGPs in the auxin efflux from cells), and signaling (the discovery of intracellular auxin receptor TIR1; modification of endocytosis by auxins and thus the control of activity of plasma membrane proteins; surfacelocalized cytokinin receptors and cytokinin signal transduction cascade, and so on). This knowledge has resulted in better understanding of the pattern formation processes and mechanisms of auxincytokinin crosstalk (shoot branching; the initiation of formation of lateral roots and their development, for example). A very valuable part of the meeting included the extremely interesting discussions between "auxin people" and "cytokinin people." Such conversations are helpful for generating new ideas, establishing new collaborations, and strengthening existing professional relationships. There was a high level of interaction between scientists and students and a free flow of information. 